\title{
Glyphosate biodegradation and potential soil bioremediation by Bacillus subtilis strain Bs-15
}

\author{
X.M. Yu' ${ }^{1}$, T. Yu ${ }^{1,2}$, G.H. Yin ${ }^{3}$, Q.L. Dong ${ }^{1,4}$, M. An ${ }^{1}$, H.R. Wang' and C.X. Ai ${ }^{1}$ \\ ${ }^{1}$ Shandong Institute of Pomology, Tai'an, Shandong, China \\ ${ }^{2}$ Shandong Agricultural University, Tai'an, Shandong, China \\ ${ }^{3}$ Department of Plant Biology and Pathology, Rutgers, \\ The State University of New Jersey, New Brunswick, NJ, USA \\ ${ }^{4}$ Research Institute of Pomology, Chinese Academy of Agricultural Sciences, \\ Xincheng, Liaoning, China \\ Corresponding author: C.X. Ai \\ E-mail: chengxiang_75@163.com
}

Genet. Mol. Res. 14 (4): 14717-14730 (2015)

Received June 23, 2015

Accepted September 15, 2015

Published November 18, 2015

DOI http://dx.doi.org/10.4238/2015.November.18.37

\begin{abstract}
Glyphosate and glyphosate-containing herbicides have an adverse effect on mammals, humans, and soil microbial ecosystems. Therefore, it is important to develop methods for enhancing glyphosate degradation in soil through bioremediation. We investigated the potential of glyphosate degradation and bioremediation in soil by Bacillus subtilis Bs-15. Bs-15 grew well at high concentrations of glyphosate; the maximum concentration tolerated by Bs- 15 reached $40,000 \mathrm{mg} / \mathrm{L}$. The optimal conditions for bacterial growth and glyphosate degradation were less than $10,000 \mathrm{mg} / \mathrm{L}$ glyphosate, with a temperature of $35^{\circ} \mathrm{C}$ and a pH of 8.0. Optimal fermentation occurred at $180 \mathrm{rpm}$ for $60 \mathrm{~h}$ with an inoculum ratio of $4 \%$. Bs-15 degraded $17.65 \%$ (12 h) to $66.97 \%$ (96 h) of glyphosate in sterile soil and 19.01\% (12 h) to 71.57\% (96 h) in unsterilized soil. Using a BIOLOG ECO plate test, we observed no significant difference in average well color development values between the soil inoculated with Bs- 15 and the control soil before $72 \mathrm{~h}$, although there was a significant difference $(\mathrm{P}<0.01)$ after $72 \mathrm{~h}$. In the presence
\end{abstract}


of Bs-15, the 5 functional diversity indices (Shannon index, Shannon uniformity, Simpson index, McIntosh index, and McIntosh uniformity) were greater $(\mathrm{P}<0.01)$ compared with the control soil. These results indicate that Bs-15 could be used to alleviate contamination from glyphosate-containing herbicides, increasing the microbial functional diversity in glyphosate-contaminated soils and thus enhancing the bioremediation of glyphosate-contaminated soils.

Key words: Bacillus subtilis; BIOLOG ECO microplate analysis; Functional diversity; Glyphosate degradation; Microbial communities; Soil bioremediation

\section{INTRODUCTION}

Glyphosate [N-(phosphonomethyl) glycine, glyphosate], a post-emergence nonselective broad-spectrum herbicide, and glyphosate-containing herbicides are the most extensively used herbicides in agriculture for the control of many annual and perennial weeds (Johal and Huber, 2009; Mbanaso et al., 2013). Weed management programs have provided highly effective weed control, simplified management decisions, and given cleaner harvested products (Johal and Huber, 2009). However, the widespread use of glyphosate may result in weed resistance or alter the biological functions of soil; additionally, glyphosate can have extensive unintended effects on nutrient availability and disease severity (Fernandez et al., 2005) resulting from direct glyphosate-induced weakening of plant defenses and increased pathogen population and virulence (Johal and Huber, 2009). Furthermore, agricultural management intensity, particularly glyphosate utilization, can alter soil microbial community structure and functional diversity. In some cases, glyphosate usage may threaten agricultural production.

Because of the impact of glyphosate and glyphosate-containing herbicides on soil microbial ecosystems and agriculture, it is important to identify methods for enhancing glyphosate degradation and biological remediation in soils. Several treatment processes are available for removing pesticides, including biodegradation, photodegradation, oxidation, flocculation and filtration, adsorption, and membrane techniques. However, glyphosate may be resistant to chemical, hydrolytic, and photolytic degradation (Romero et al., 2011).

Many studies have shown that glyphosate can be degraded by microorganisms and plants. The most active glyphosate-degrading microorganisms were isolated from soils polluted by organophosphonates (Shushkova et al., 2010). In soil, glyphosate is primarily decomposed by bacteria and fungi, which utilize glyphosate as carbon source, leading to the production of aminomethylphosphonic acid, or as a phosphorus source, producing glycine (Solomon et al., 2007). Bacteria are perhaps the most versatile and diversified organisms with regard to their nutritional requirements (Leckie, 2005). The development of an affordable and environmentally friendly bioremediation method using glyphosate-degrading bacteria is a promising approach for cleansing and restoring soils contaminated with this herbicide (Kryuchkova et al., 2014).

Plant growth promotion rhizobacteria are free-living soil bacteria that can directly or indirectly facilitate plant growth and control plant diseases under optimal conditons and biotic and abiotic stresses. Although plant growth promotion rhizobacteria are commonly used to promote plant growth and control plant diseases, their potential use in bioremediation has also been explored (Narasimhan et al., 2003; Huang et al., 2005). Several studies have been 
conducted to isolate pure bacterial strains with degrading capability for potential uses such as the removal of glyphosate from soil and bioremediation of glyphosate-contaminated soils (Ermakova et al., 2008; Ahire et al., 2012; Kryuchkova et al., 2014).

The Bacillus subtilis strain Bs-15 (initially named CAS15) was isolated from the rhizospheric soil of a pepper plant, and it has shown significant potential for plant growth promotion and the biological control of plant disease (Yu et al., 2010, 2011). The objective of this study was to investigate the glyphosate degradation capacity of $B$. subtilis Bs- 15 and its potential use for the bioremediation of soil polluted with organophosphorus compounds.

\section{MATERIAL AND METHODS}

\section{Reagents}

Analytical-standard amine-glyphosate (99.2\%) was obtained from the Shanghai Pesticide Research Institute (Shanghai, China), analytical reagent-grade disodium tetraborate decahydrate $\left(\mathrm{Na}_{2} \mathrm{~B}_{4} \mathrm{O}_{7} \cdot 10 \mathrm{H}_{2} \mathrm{O}\right)$ was purchased from Sigma (St. Louis, MO, USA), and 9-fluorenyl methoxycarbonyl chloride (FMOC-Cl) was purchased from Merck (White House Station, NJ, USA). Acetonitrile and trichloromethane were of high-performance liquid chromatography grade. All other chemicals $\left(\mathrm{KCl}, \mathrm{HCl}, \mathrm{KOH}, \mathrm{Fe}\left(\mathrm{NO}_{3}\right)_{3} \cdot 9 \mathrm{H}_{2} \mathrm{O}\right.$, and $\left.\mathrm{NaOH}\right)$ were analyticalgrade. Borate buffer, $\mathrm{pH} 9.0$, was prepared by dissolving $1.5255 \mathrm{~g} \mathrm{Na}_{2} \mathrm{~B}_{4} \mathrm{O}_{7} \cdot 10 \mathrm{H}_{2} \mathrm{O}$ in $100 \mathrm{~mL}$ ultrapure water. The FMOC-Cl solutions were prepared by dissolving the reagent in acetonitrile to a concentration of $1 \mathrm{~g} / \mathrm{L}$. Fresh FMOC-Cl solutions were prepared immediately before each experiment.

\section{Acclimation of $B$. subtilis Bs-15}

The B. subtilis strain Bs-15 was isolated from the rhizospheric soil of a pepper plant. This strain has shown significant potential for plant growth promotion and the biological control of plant disease (Yu et al., 2010, 2011). In this study, B. subtilis strain Bs-15 was first acclimated in a mineral MS1 medium (Ermakova et al., 2008) containing different concentrations of glyphosate. All salts were of "particularly high-purity" or "chemical purity" grade. For consistency under the following test conditions, glyphosate was used as the carbon and phosphorus source. The initial culture plating density for the first acclimation step $(25 \mathrm{mg} / \mathrm{L})$ was $\mathrm{OD}_{600}=0.6-0.8$, suggesting that bacteria were in the exponential growth phase. Next, 2-day surviving cultures were inoculated into mineral MS1 medium with higher concentrations of glyphosate. Bs-15 was subcultured in mineral MS1 medium containing the following concentrations of glyphosate: 25, 50, 100, 200, 400, 1000, 2000, 4000, and 40,000 mg/L. Bs-15 grows well at high concentrations of glyphosate, with a maximum tolerance of $40,000 \mathrm{mg} / \mathrm{L}$.

\section{Glyphosate quantification}

Glyphosate was quantified by UV-vis spectrophotometry (Waiman et al., 2012) after derivatization with $\mathrm{FMOC}-\mathrm{Cl}$ in alkaline media, using a protocol adopted from Vreeken et al. (1998) and Le Fur et al. (2000). Aqueous glyphosate solutions were prepared in $0.1 \mathrm{M} \mathrm{KCl}$, which was used as the supporting electrolyte. Next, $0.5 \mathrm{~mL}$ borate buffer was mixed with 3 $\mathrm{mL}$ glyphosate supernatant in $15 \mathrm{~mL}$ polypropylene centrifuge tubes. Next, $0.5 \mathrm{~mL}$ FMOC- 
$\mathrm{Cl}$ solution was added and the mixture was homogenized by manual shaking. After $2 \mathrm{~h}$ at room temperature, the resulting solution was mixed with $4 \mathrm{~mL}$ trichloromethane, shaken, and centrifuged at $4000 \mathrm{rpm}$ for $6 \mathrm{~min}$ to separate the trichloromethane from the water. Trichloromethane was used to extract excess derivatization reagent $(\mathrm{FMOC}-\mathrm{Cl})$ to prevent interference with spectrophotometric measurements. The aqueous phase containing the derivatization product was removed and quantified by UV-vis spectrophotometry using an Ultrospc 2100 pro UV-Visible spectrophotometer (Amersham Biosciences, Amersham, UK) equipped with $1-\mathrm{cm}$ quartz cuvettes.

Because UV-vis spectrophotometry can only quantify glyphosate concentrations ranging from 0.084-21.8 $\mathrm{mg} / \mathrm{L}$ (Waiman et al., 2012), glyphosate solutions were diluted to concentrations of $\leq 21.8 \mathrm{mg} / \mathrm{L}$. Full-wavelength scanning $(200-999 \mathrm{~nm})$ showed that derivatized glyphosate has a maximum absorbance at $265 \mathrm{~nm}$, which is identical to the detection wavelength described by Waiman et al. (2012). Thus, $265 \mathrm{~nm}$ was used for glyphosate quantification. In all cases, a blank solution was prepared by combining $3 \mathrm{~mL}$ pure $0.1 \mathrm{M} \mathrm{KCl}$ with $0.5 \mathrm{~mL}$ borate buffer, $0.5 \mathrm{~mL}$ FMOC-Cl, and $4 \mathrm{~mL}$ trichloromethane, and then shaking and centrifugation as described above.

\section{Detection of glyphosate degradation by Bs-15}

Bs-15 was inoculated into $50 \mathrm{~mL}$ mineral MS1 medium containing 10,000 mg/L glyphosate in $150-\mathrm{mL}$ conical flasks. The cultures were incubated at $30^{\circ} \mathrm{C}$ with shaking at 150-200 rpm. Every $12 \mathrm{~h}, 5 \mathrm{~mL}$ bacterial culture was removed to determine the rate of bacterial growth situation and glyphosate degradation. After centrifugation at 12,000 rpm for $5 \mathrm{~min}$, the supernatant was filtered through a $0.22-\mu \mathrm{m}$ filter. The filtered supernatant was adjusted to $0.1 \mathrm{M} \mathrm{KCl}$ and then treated as described above. Glyphosate content was quantified by UV-vis spectrophotometry at $265 \mathrm{~nm}$. The positive control contained the same volume of mineral MS1 medium with $5000 \mathrm{mg} / \mathrm{L}$ glyphosate, while the blank solution contained $3 \mathrm{~mL}$ pure 0.1 $\mathrm{M} \mathrm{KCl}$. To ensure accuracy and precision, the aqueous phase was diluted to less than 21.8 $\mathrm{mg} / \mathrm{L}$ before quantification, and each treatment was repeated 3 times. The degradation rate was calculated using the following formula to generate the degradation curve:

$$
\text { Degradation rate }(\%)=\left(M_{2}-M_{1}\right) / M_{2} \times 100 \%
$$

where $M_{1}$ is the quantity of glyphosate in the treated sample and $M_{2}$ is the quantity of glyphosate in the positive control.

\section{Factors influencing glyphosate degradation}

Mineral MS1 medium was used to identify factors influencing glyphosate degradation by Bs- 15 . The following factors were investigated: initial glyphosate concentration (2500, $5000,10,000,20,000$, and $40,000 \mathrm{mg} / \mathrm{L})$; temperature $\left(20^{\circ}, 25^{\circ}, 30^{\circ}, 35^{\circ}\right.$, and $\left.40^{\circ} \mathrm{C}\right)$; rotation speed $(90,120,150,180$, and $210 \mathrm{rpm})$; initial $\mathrm{pH}$ value of the medium $(5.0,6.0,7.0,8.0$, and 9.0); inoculum ratio $(2,4,6,8$, and $10 \%)$; exogenous mineral salts $\left(\mathrm{MgSO}_{4}, \mathrm{NaCl}, \mathrm{KCl}\right.$, and $\mathrm{CaCl}_{2}$ ), using medium without exogenous mineral salt as a control; exogenous carbon sources (barley sugar, fructose, glucose, sucrose, and mannitol), using medium without an exogenous carbon source as a control; and exogenous nitrogen sources (yeast, soy peptone, tryptone, 
peptone, and beef extract), using medium without an exogenous nitrogen source as a control. Each treatment was repeated 3 times and degradation rate was calculated as described above. The mineral MS1 medium optimized for Bs-15 growth and glyphosate degradation was used for the following experiments.

\section{Degradation of glyphosate in soil}

The degradation of glyphosate in the soil by Bs- 15 was assayed under artificially controlled conditions. First, soil samples were collected from organic vegetable fields. After removing the rough plant residues and sieving, $50 \mathrm{~g}$ soil was added to $250-\mathrm{mL}$ conical flasks. To facilitate diluting the glyphosate concentrations to less than $21.8 \mathrm{mg} / \mathrm{L}$, the initial glyphosate concentration used in soil experiments was $5000 \mathrm{mg} / \mathrm{L}$. Four treatment groups were used as follows: 1$)$ a suspension $\left(\mathrm{OD}_{600}=0.6-0.8\right)$ of Bs-15 cultured in the optimized mineral MS1 medium containing $5000 \mathrm{mg} / \mathrm{L}$ glyphosate was inoculated into sterile soil (which was previously autoclaved at $121^{\circ} \mathrm{C}$ for $2 \mathrm{~h}$ ); 2) optimized mineral MS1 medium containing $5000 \mathrm{mg} / \mathrm{L}$ glyphosate was inoculated into sterile soil; 3) a suspension of Bs-15 cultured in optimized mineral MS1 medium containing $5000 \mathrm{mg} / \mathrm{L}$ glyphosate was inoculated into unsterilized soil; and 4) optimized mineral MS1 medium containing $5000 \mathrm{mg} / \mathrm{L}$ glyphosate was inoculated into unsterilized soil. The solutions were mixed until they were homogenous, after which the conical flasks were incubated at $30^{\circ} \mathrm{C}$. Every $6 \mathrm{~h}, 2.0 \mathrm{~g}$ treated soil was removed for glyphosate quantification. Each treatment was repeated 3 times.

For the soil samples, isotherms were determined by weighing $2.0 \mathrm{~g}$ soil into $15-\mathrm{mL}$ polypropylene centrifuge tubes. Next, $0.1 \mathrm{M} \mathrm{KCl}$ and glyphosate were added to obtain a concentration range from 7-190 mg/L. The final volume in each tube was brought to $10 \mathrm{~mL}$. Either $\mathrm{NaOH}$ or $\mathrm{HCl}$ solutions were added to standardize the $\mathrm{pH}$. The tubes were equilibrated by shaking overnight. The tubes were then centrifuged at $4000 \mathrm{rpm}$ for $10 \mathrm{~min}$, and the supernatants were removed for derivatization and further analysis. The amount of degraded glyphosate was calculated based on the difference between the initial glyphosate concentration and the concentration of the herbicide remaining in the supernatant. Isotherm determinations were performed at $\mathrm{pH} 4.5,6.0$, and 8.0.

\section{Analysis of functional diversity of soil microbes}

The functional diversity of the soil microbial communities was measured using the BIOLOG method (Garland and Mills, 1991; Stefanowicz, 2006; Sun et al., 2012; Li et al., 2013). Five grams of fresh soil containing $5000 \mathrm{mg} / \mathrm{L}$ glyphosate with and without Bs- 15 was suspended in $50 \mathrm{~mL} 0.85 \%$ sterile $\mathrm{NaCl}$ solution, shaken for $30 \mathrm{~min}$ on a reciprocal shaker, and diluted 200 -fold. Next, $150-\mu \mathrm{L}$ aliquots of diluted samples were inoculated directly into ECO microplates (BIOLOG, Hayward, CA, USA) and incubated at $25^{\circ} \mathrm{C}$ in the dark without shaking. Color development was measured spectrophotometrically (Kelly and Tate, 1998), and the plates were read every $24 \mathrm{~h}$ at $590 \mathrm{~nm}$ for $168 \mathrm{~h}$ using the Microlog Rel 4.2 software.

Average well color development (AWCD) was calculated for each microplate using the following equation: $\mathrm{AWCD}=\Sigma(A j-A k) / 31$, where $A j$ is raw absorbance in the well $j$, and $A k$ is the absorbance in control well $A 1$ (Garland and Mills, 1991). The functional diversity parameters of the microbial communities were calculated as described by Garland and Mills (1991) using the AWCD values from the $96 \mathrm{~h}$ time point, as these values represented approxi- 
mately $50 \%$ saturation where the highest differentiation was expected (Garland and Mills, 1991; Li et al., 2007).

Before statistical analysis, the absorbance value of a control well (no substrate) was subtracted from the absorbance value of each well containing a substrate to avoid negative absorbance values (Hitzl et al., 1997).

All results are reported based on oven-dry soil weight. All statistical analyses were conducted using the SPSS 17.0 software (SPSS Inc., Chicago, IL, USA). Both treatments were repeated 3 times. The means and standard deviations were compared by one-way analysis of variance, and significant differences were analyzed by Duncan's method (SAS Institute, Cary, NC, USA).

\section{RESULTS AND DISCUSSION}

\section{B. subtilis Bs-15 survival in glyphosate}

Moneke et al. (2010) demonstrated that bacterial genera that were endemic in soils and often present in pervious paving systems, such as Pseudomonas, Azotobacter, Acetobacter, and Alcaligenes, could grow on mineral media using glyphosate as the sole carbon source. This study showed that survival of $B$. subtilis Bs-15 decreased with increasing glyphosate concentration. When glyphosate concentration reached $40,000 \mathrm{mg} / \mathrm{L}, \mathrm{OD}_{600}=0.165$, while the $\mathrm{OD}_{600}$ value of the culture liquid without glyphosate was 0.037 , indicating that Bs- 15 survived in $40,000 \mathrm{mg} / \mathrm{L}$ glyphosate and that Bs-15 could use glyphosate as carbon and phosphate sources. Thus, we concluded that Bs- 15 could tolerate up to $40,000 \mathrm{mg} / \mathrm{L}$ glyphosate in culture by successive subculturing.

\section{Glyphosate degradation curve}

After inoculation of Bs-15 into glyphosate-containing mineral MS1 medium, derivatization with $\mathrm{FMOC}-\mathrm{Cl}$ in alkaline media and quantification of glyphosate via UV-vis spectrophotometry, a degradation curve of glyphosate was obtained. Bs-15 growth declined rapidly and the rate of glyphosate degradation increased rapidly up to $48 \mathrm{~h}$. After the growth and degradation stabilized, no significant differences were observed between 60 and $72 \mathrm{~h}$ (Figure 1). Thus, the optimal incubation time was $60 \mathrm{~h}$.

Highly sensitive analytical techniques for glyphosate quantification have been developed in recent years; however, most of the methods are complex and require expensive, specialized equipment that is not economically feasible for many laboratories. Furthermore, these methods allow glyphosate to be quantified at low levels in natural samples. While glyphosate applied in bioremediation is typically applied to soils in the form of aqueous solutions at approximately $0.03 \mathrm{M}$ (Candela et al., 2010) or higher (Laitinen et al., 2009), these high concentrations are also environmentally relevant. Any investigation of glyphosate should address these high concentrations. Therefore, it is necessary to use simple, rapid, and low-cost methods for glyphosate quantification, although the methods are expected to be less sensitive than chromatography, capillary electrophoresis, or other methods.

Some researchers have attempted to use UV-vis spectroscopy to quantify glyphosate. Glass (1981) proposed a colorimetric method based on the oxidation of glyphosate to orthophosphate with hydrogen peroxide. Although this method effectively quantified glyphosate 
concentrations from $1-20 \mathrm{mg} / \mathrm{L}$, it is hazardous, as hydrogen peroxide occasionally causes explosions during the evaporation step. Based on this, Waiman et al. (2012) proposed a simple, rapid, and low-cost UV-vis spectrophotometric method to quantify glyphosate. This method includes a derivatization step and further measurement of the absorbance at the appropriate UV wavelength, and it can be used to perform adsorption isotherms on soils and metal oxides.

The spectrophotometric method proposed by Waiman et al. (2012) can quantify glyphosate concentrations ranging from $0.084-21.8 \mathrm{mg} / \mathrm{L}$. The advantages of this method are its simplicity, rapidity, and low cost of analysis. The results obtained using this method did not differ significantly from those obtained using ultra-high-performance liquid chromatography with tandem mass spectrometry detection. Therefore, this simple and low-cost method for glyphosate quantification can be used in certain applications, although they are less sensitive than chromatography or other methods.

We used the method proposed by Waiman et al. (2012) to quantify glyphosate in Bs15 cultures. Our results were repeatable, and glyphosate degradation corresponded to the rate of Bs-15 growth, indicating that this quantification method was reliable.

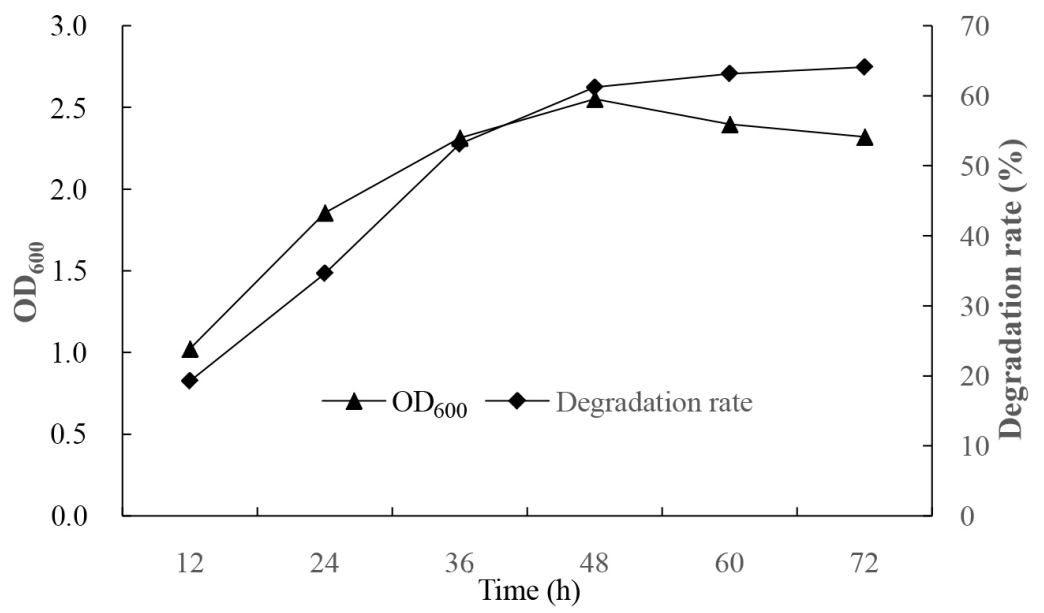

Figure 1. Curve of Bs-15 growth and glyphosate degradation.

\section{Factors influencing glyphosate degradation}

Multiple factors that may influence glyphosate degradation by Bs-15, including temperature, rotation speed, initial $\mathrm{pH}$ value, inoculum ratio, initial glyphosate concentration, different species of inorganic salts, carbon sources, and nitrogen sources, were analyzed. The optimal conditions for bacterial growth and glyphosate degradation were less than 10,000 $\mathrm{mg} / \mathrm{L}$ glyphosate, with a degradation rate of approximately $65 \%$, a temperature of $35^{\circ} \mathrm{C}$, a $\mathrm{pH}$ of 8.0, and fermentation occurred at $180 \mathrm{rpm}$ for $60 \mathrm{~h}$ with an inoculum ratio of $4 \%$ (Figure 2). The optimal additional inorganic salt, carbon source, and nitrogen source for glyphosate degradation were $\mathrm{MgSO}_{4}$, fructose, and yeast, respectively (Figure 3).

Although the optimal temperature for bacterial growth and glyphosate was $35^{\circ} \mathrm{C}$ in pure cultures, this is not realistic temperature for field settings, and thus the incubation temperature used was $30^{\circ} \mathrm{C}$. 

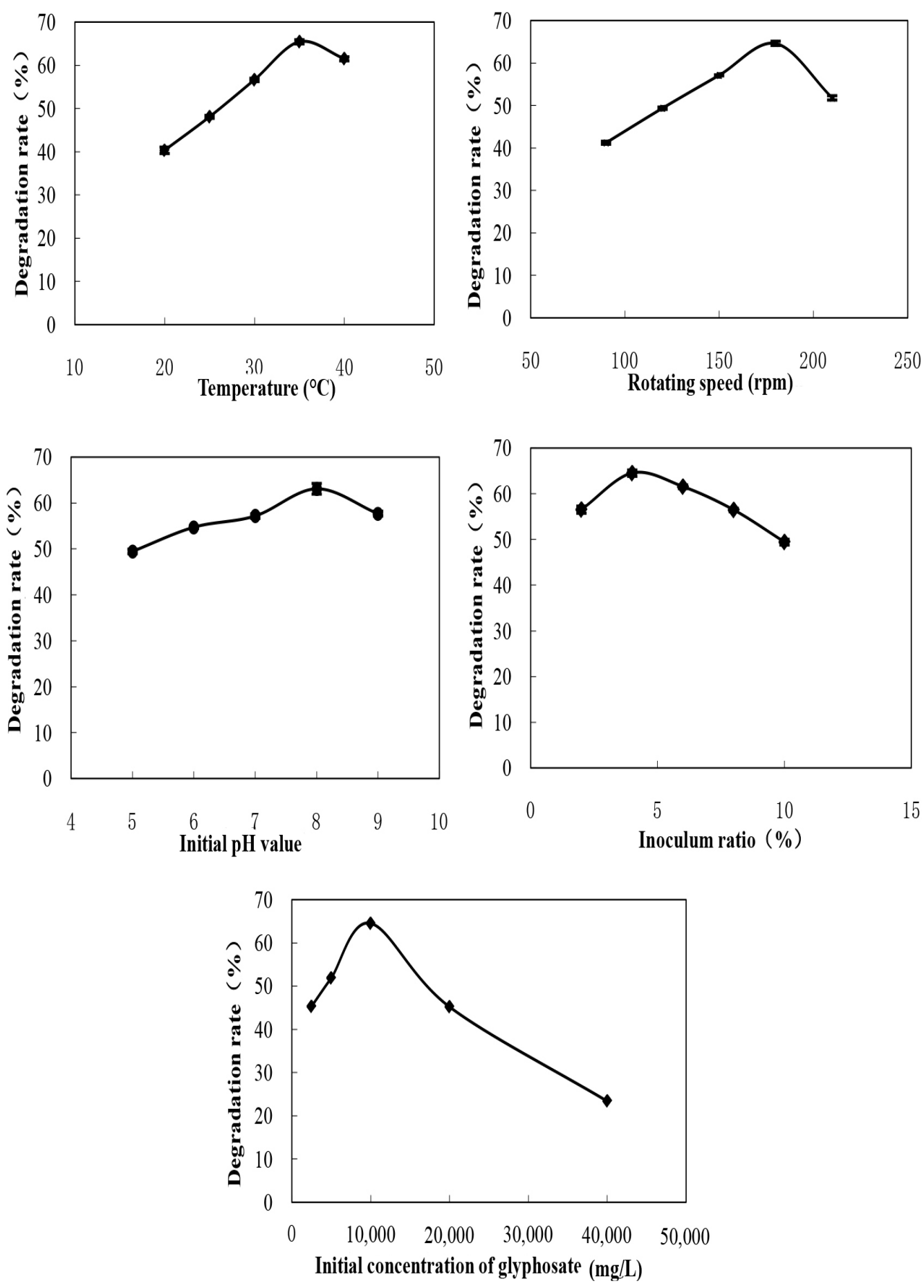

Figure 2. Inoculation factors influence the glyphosate degradation by Bs-15. 


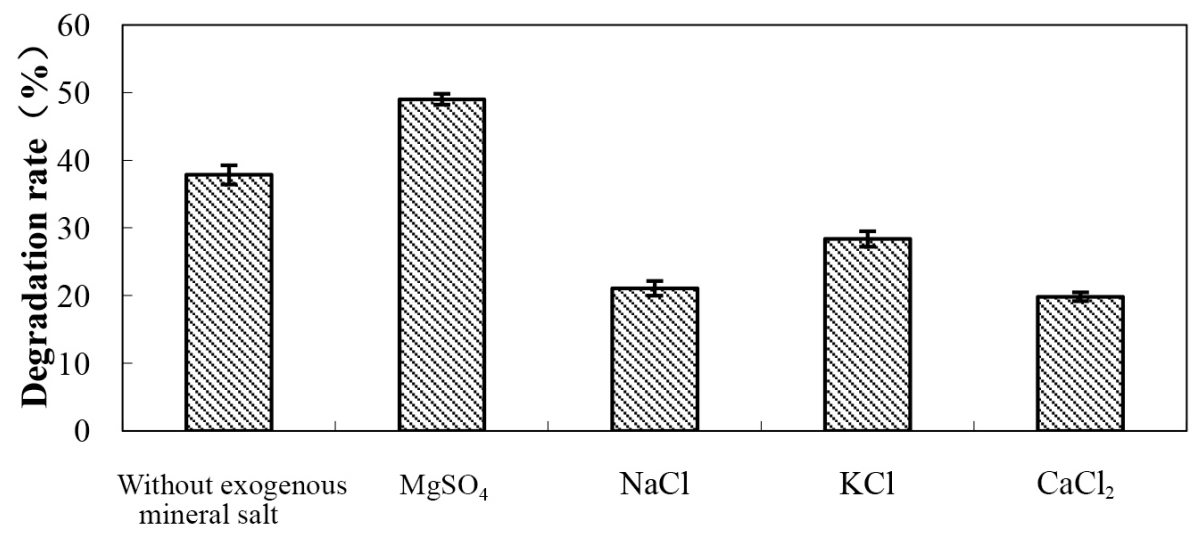

Additional inorganic salt

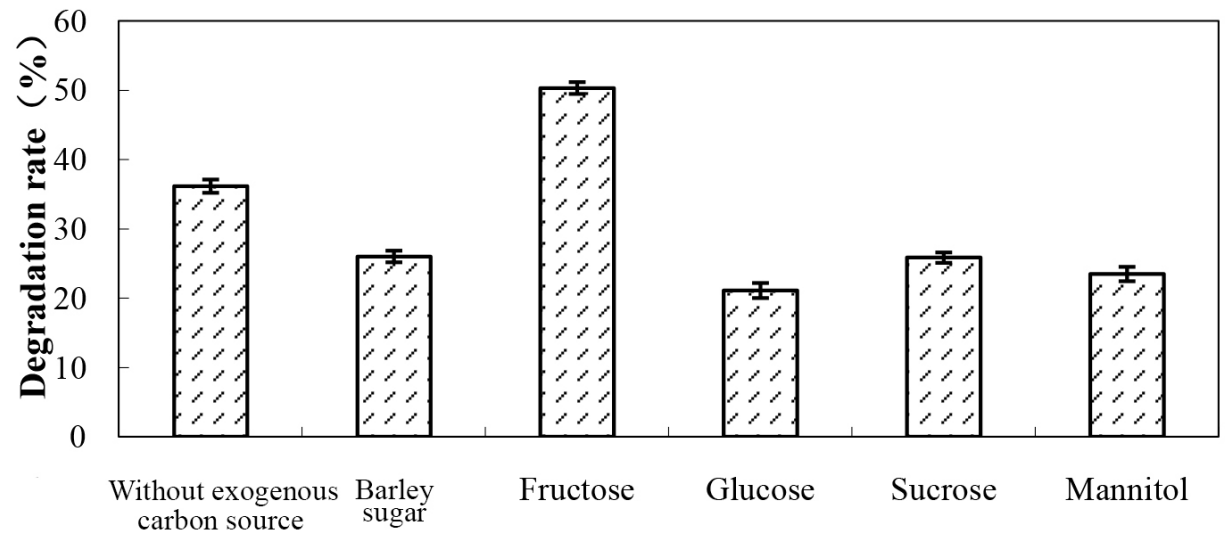

Additional carbon source

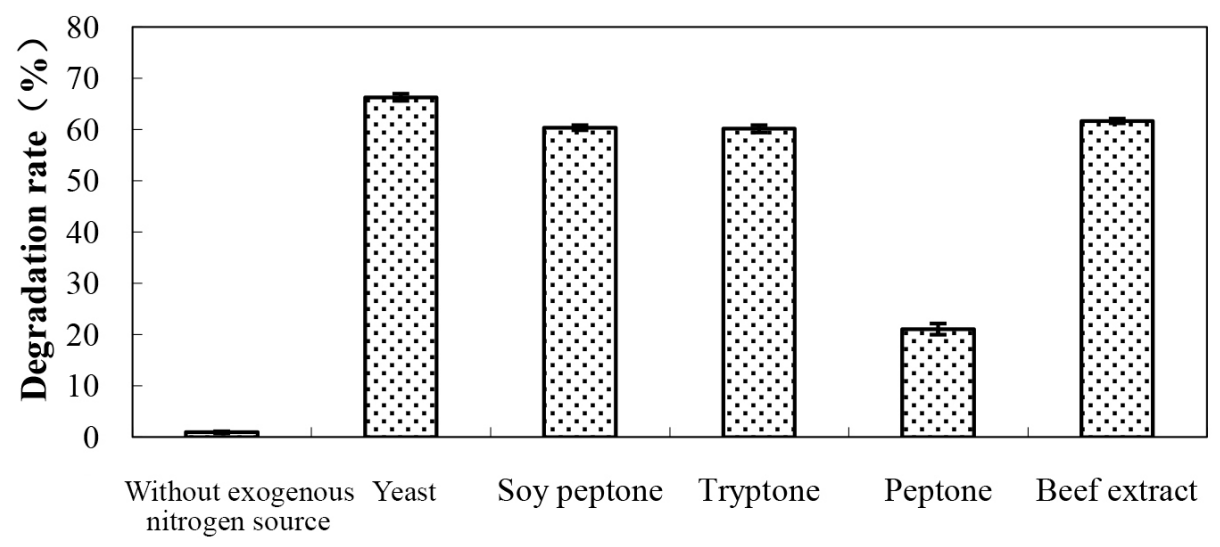

\section{Additional nitrogen source}

Figure 3. Additional sources influence the glyphosate degradation by Bs-15. 
When Bs-15 was inoculated into glyphosate-containing optimized mineral MS1 medium and incubated under the optimized conditions, the rate of glyphosate degradation was 66.35, which was $5.07 \%$ greater than the degradation rate in mineral MS1 medium (Figure 1). These results demonstrate that optimization of exogenous factors enhanced the degradation of glyphosate by Bs- 15 .

\section{Bs-15-mediated biodegradation of glyphosate in soil}

We observed a greater degree of glyphosate biodegradation in unsterilized soil than in sterile soil. The glyphosate concentration in sterile soil and unsterilized soil without Bs-15 decreased stably over time, while the glyphosate concentration in the sterilized soil containing Bs-15 decreased rapidly up to $30 \mathrm{~h}$ and then slowly up to $60 \mathrm{~h}$. After $60 \mathrm{~h}$, the rate of glyphosate degradation in the sterilized soil containing Bs-15 was similar to that in the soil without Bs- 15 , and the degradation trends were very similar. The degradation of glyphosate increased over time, with the degradation rate by Bs-15 in sterile soil ranging from $17.65 \%$ (12 h) to $66.97 \%(96 \mathrm{~h})$, and the degradation rate in unsterilized soil ranging from $19.01 \%(12 \mathrm{~h})$ to $71.57 \%$ (96 h) (Figure 4). These results indicate that there are microorganisms other than Bs15 that help to degrade glyphosate in natural soil (Patil et al., 1970).

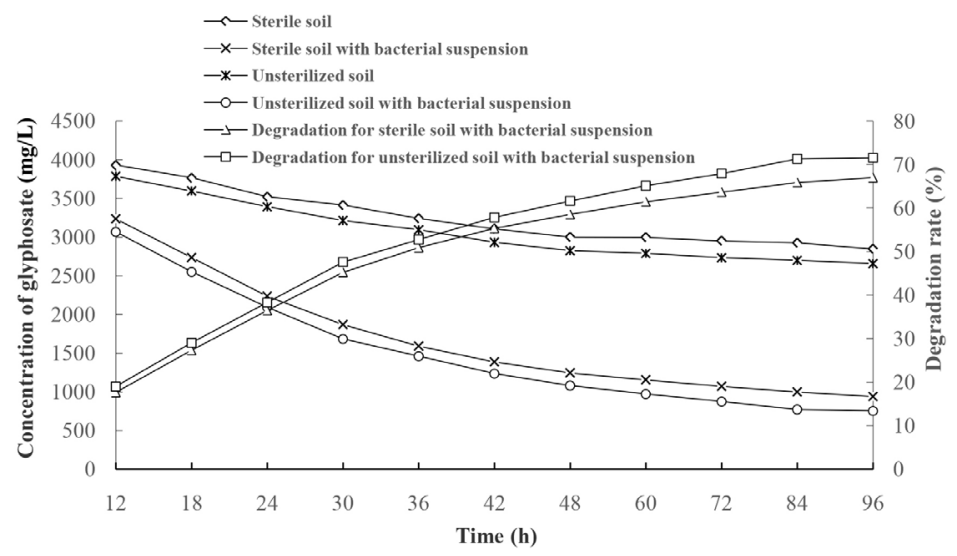

Figure 4. Degradation of glyphosate in soil by Bs- 15 .

Glyphosate is rapidly degraded in soils: up to $20-70 \%$ of glyphosate is mineralized to $\mathrm{CO}_{2}$ over approximately 5 weeks, depending on the soil type (Zablotowicz et al., 2009; Ding et al., 2011). We observed degradation rates of glyphosate by Bs- 15 in unsterilized soil ranging from 19.01-71.57\% within 1 week and degradation rates in sterile soil ranging from 17.65$66.97 \%$. Our results indicate that Bs-15 significantly enhanced glyphosate degradation in soil.

\section{Changes in the functional diversity of soil microbial communities induced by Bs-15}

In the BIOLOG ECO plate assay, we observed that AWCD values increased over time. The AWCD values from the soils inoculated with Bs- 15 increased rapidly up to $72 \mathrm{~h}$ and continued to increase at a slower rate up to $120 \mathrm{~h}$, while the AWCD values from the control soil increased steadily. There was no significant difference between AWCD values in the treat- 
ment group (inoculated with Bs-15) and control soil (without Bs-15 inoculation) before $72 \mathrm{~h}$, but there was a significant difference $(\mathrm{P}<0.01)$ after $72 \mathrm{~h}$ (Figure 5).

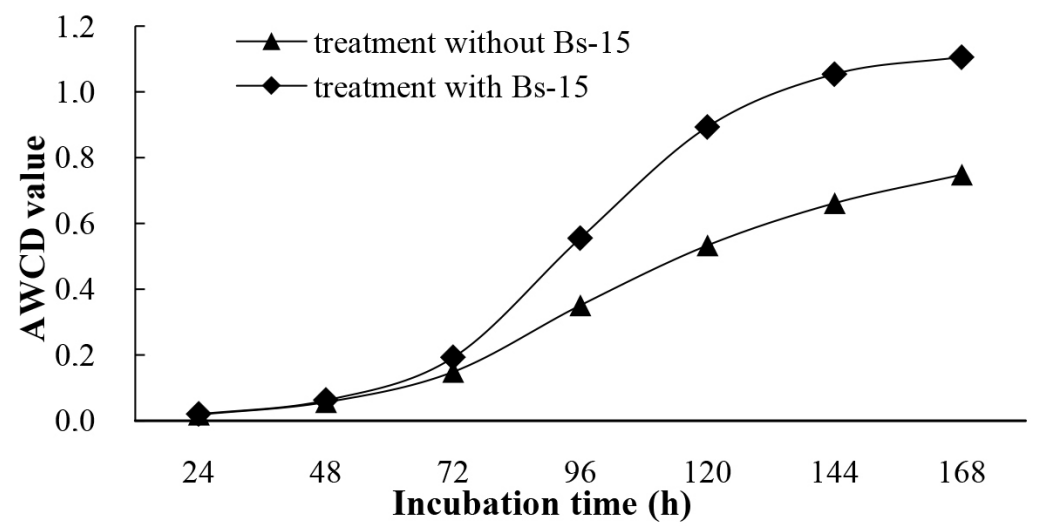

Figure 5. Change in average well color development (AWCD) of soil microbial communities by Bs-15.

Changes in the functional diversity of soil microbial communities are reflected by 3 functional diversity indices. The Shannon index and Shannon uniformity are strongly influenced by the species richness of communities, the Simpson index reflects the most common species, and the McIntosh index and McIntosh uniformity measure the uniformity of species (Atlas, 1984; Maguran, 1988).

The BIOLOG values from the $96 \mathrm{~h}$ time point were used to calculate the functional diversity indices of the microbial communities. In the presence of Bs-15 application, the 5 functional diversity indices (Shannon index, Shannon uniformity, Simpson index, McIntosh index, and McIntosh uniformity) showed significant differences $(\mathrm{P}<0.01)$ compared to the control sample (Table 1).

Table 1. Changes in functional diversity indexes of soil microbial communities by Bs- 15.
\begin{tabular}{lccccc}
\hline Treatment & Shannon index & Shannon uniformity & Simpson index & McIntosh index & McIntosh uniformity \\
\hline CK (soil without Bs-15 inoculation) & $4.139 \pm 0.020^{\mathrm{Bb}}$ & $0.940 \pm 0.004^{\mathrm{Bb}}$ & $77.815 \pm 0.664^{\mathrm{Bb}}$ & $3.156 \pm 0.029^{\mathrm{Bb}}$ & $0.959 \pm 0.004^{\mathrm{Bb}}$ \\
Soil with Bs-15 inoculation & $4.401 \pm 0.011^{\mathrm{Aa}}$ & $0.976 \pm 0.005^{\mathrm{Aa}}$ & $81.788 \pm 0.678^{\mathrm{Aa}}$ & $3.370 \pm 0.038^{\mathrm{Aa}}$ & $0.992 \pm 0.004^{\mathrm{Aa}}$ \\
Increase rate (\%) & 6.33 & 3.83 & 5.11 & 6.79 & 3.37 \\
\hline
\end{tabular}

Results are means \pm SE. Values within a column followed with different superscript lowercase letters are significantly different at $\mathrm{P}<0.05$ level, with different superscript capital letters are significantly different at $\mathrm{P}<0.01$ level.

Analysis of the functional biodiversity of soil microbial communities yields important information regarding the biological quality and fertility of the soil. Functional diversity is considered more ecologically relevant than taxonomic diversity, and it is typically measured using the BIOLOG system (Stefanowicz, 2006; Li et al., 2013). The BIOLOG ECO microplates are designed for ecological studies of whole microbial communities and are typically used in comparative studies, such as for comparing the functional diversity of microbial communities from contaminated and non-contaminated soils (Stefanowicz, 2006).

The most significant advantage of the BIOLOG ECO microplates is that they contain 3 replicates of each of the 31 substrates and a control well with no substrate (Preston-Mafham 
et al, 2002; Classen et al., 2003). The BIOLOG method facilitates the observation of even small shifts in microbial communities, and thus the BIOLOG plates technique is extremely useful for environmental protection and can be used as a rapid method for estimating changes in soil microbial communities in response to short or long-term exposure to contamination. Furthermore, this method enables the evaluation of adverse changes in microbial communities based on differences in metabolic responses between communities from contaminated and control areas (Stefanowicz, 2006).

The BIOLOG method is sensitive and rapid. In general, greater biodiversity is positive, as it stabilizes ecosystem functions (Atlas, 1984), and a reduction in biodiversity often indicates the extinction of sensitive species as a result of contamination (Rutgers et al., 1998).

Our BIOLOG ECO microplate test results showed that in the presence of Bs-15, the 5 functional diversity indices of soil microbial communities increased significantly $(\mathrm{P}<0.01)$ compared to the control soil. This suggests that Bs- 15 may help to alleviate contamination by glyphosate-containing herbicides and enhance microbial functional diversity in glyphosatecontaminated soil.

\section{CONCLUSIONS}

We have previously shown that $B$. subtilis Bs- 15 promotes plant growth and controls plant diseases. In this study, we investigated the degradation of glyphosate and soil bioremediation by Bs- 15 . We observed that Bs- 15 grew well at high concentrations of glyphosate and could tolerate glyphosate concentrations up to $10,000 \mathrm{mg} / \mathrm{L}$. Bs- 15 has a high capacity for glyphosate degradation and degraded approximately $65 \%$ of $10,000 \mathrm{mg} / \mathrm{L}$ glyphosate under optimal fermentation conditions. The AWCD values from the BIOLOG ECO microplate test and the 5 functional diversity indices (Shannon index, Shannon uniformity, Simpson index, McIntosh index, and McIntosh uniformity) were significantly different $(\mathrm{P}<0.01)$ between the group inoculated with Bs- 15 and the control group. These results indicate that Bs- 15 can significantly promote glyphosate degradation in soil and play an important role in the bioremediation of glyphosate-contaminated soils.

The results of this study and our previous study indicate that B. subtilis Bs- 15 is a promising bio-inoculant for plant growth promotion, biological control of plant disease, and glyphosate degradation, and it is useful for the bioremediation of soils polluted with difficultto-hydrolyze organophosphorus chemicals.

\section{ACKNOWLEDGMENTS}

Research supported by the National Natural Science Foundation of China (Grant \#31100086).

\section{REFERENCES}

Ahire KC, Kapadnis BP, Kulkarni GJ, Shouche YS, et al. (2012). Biodegradation of tributyl phosphate by novel bacteria isolated from enrichment cultures. Biodegradation 23: 165-176.

Atlas RM (1984). Diversity of microbial community. Adv. Microbiol. Ecol. 7: 19-47.

Candela L, Caballero J and Ronen D (2010). Glyphosate transport through weathered granite soils under irrigated and non-irrigated conditions - Barcelona, Spain. Sci. Total Environ. 408: 2509-2516.

Classen AT, Boyle SI, Haskins KE, Overby ST, et al. (2003). Community-level physiological profiles of bacteria and fungi: plate type and incubation temperature influences on contrasting soils. FEMS Microbiol. Ecol. 44: 319-328. 
Ding W, Reddy KN, Zablotowicz RM, Bellaloui N, et al. (2011). Physiological responses of glyphosate-resistant and glyphosate-sensitive soybean to aminomethylphosphonic acid, a metabolite of glyphosate. Chemosphere 83: 593-598.

Ermakova IT, Shushkova TV and Leont'evskiĭ AA (2008). Microbial degradation of organophosphonates by soil bacteria. Mikrobiologiia 5: 689-695.

Fernandez MR, Selles F, Gehl D, DePauw RM, et al. (2005). Crop production factors associated with Fusarium head blight in spring wheat in eastern Saskatchewan. Crop Sci. 45: 1908-1916.

Garland JL and Mills AL (1991). Classification and characterization of heterotrophic microbial communities on the basis of patterns of community-level-sole-carbon-source utilization. Appl. Environ. Microbiol. 57: 2351-2359.

Glass RL (1981). Colorimetric determination of glyphosate in water after oxidation to orthophosphate. Anal. Chem. 53: 921-923.

Hitzl W, Rangger A, Sharma S and Ininsam H (1997). Separation power of 95 substrates of the Biolog system determined in various soils. FEMS Microbiol. Ecol. 22: 167-174.

Huang XD, El-Alawi Y, Gurska J, Glick BR, et al. (2005). A multi-process phytoremediation system for decontamination of persistent total petroleum hydrocarbons (TPHs) from soils. Microchem. J. 81: 139-147.

Johal GS and Huber DM (2009). Glyphosate effects on diseases of plants. Eur. J. Agron. 31: 144-152.

Kelly JJ and Tate RL (1998). Use of Biolog for the analysis of microbial communities from zinc-contaminated soils. $J$. Environ. Qual. 27: 609-617.

Kryuchkova YV, Burygin GL, Gogoleva NE, Gogolev YV, et al. (2014). Isolation and characterization of a glyphosatedegrading rhizosphere strain, Enterobacter cloacae K7. Microbiol. Res. 169: 99-105.

Laitinen P, Rämö S, Nikunen U, Jauhiainen L, et al. (2009). Glyphosate and phosphorus leaching and residues in boreal sandy soil. Plant Soil 323: 267-283.

Le Fur E, Colin R, Charrêteur C, Dufau C, et al. (2000). Determination of glyphosate herbicide and aminomethylphosphonic acid in natural waters by liquid chromatography using pre-column fluorogenic labeling. Part I: direct determination at the $0.1 \mathrm{mg} / \mathrm{L}$ level using FMOC. Analusis 28: 813-818.

Leckie SE (2005). Methods of microbial community profiling and their application to forest soils. Forest Ecol. Manag. 220: 88-106.

Li FL, Liu M, Li ZP, Jiang CY, et al. (2013). Changes in soil microbial biomass and functional diversity with a nitrogen gradient in soil columns. App. Soil Ecol. 34: 1-6.

Li ZP, Wu XC and Chen BY (2007). Changes in transformation of soil organic C and functional diversity of soil microbial community under different land uses. Agric. Sci. China 6: 1235-1245.

Maguran AE (1988). Ecological diversity and its measurement. Princeton University Press, Princeton, 141-162.

Mbanaso FU, Coupe SJ, Charlesworth SM and Nnadi EO (2013). Laboratory-based experiments to investigate the impact of glyphosate-containing herbicide on pollution attenuation and biodegradation in a model pervious paving system. Chemosphere 90: 737-746.

Moneke AN, Okpala GN and Anyanwu CU (2010). Biodegradation of glyphosate herbicide in vitro using bacterial isolates from four rice fields. Afr. J. Biotechnol. 9: 4067-4074.

Narasimhan K, Basheer C, Bajic VB and Swarup S (2003). Enhancement of plant-microbe interactions using a rhizosphere metabolomics-driven approach and its application in the removal of polychlorinated biphenyls. Plant Physiol. 132: 146-153.

Patil KC, Matsumura F and Boush GM (1970). Degradation of endrin, aldrin, and DDT by soil microorganisms. Appl. Microbiol. 19: 879-881.

Preston-Mafham J, Boddy L and Randerson PF (2002). Analysis of microbial community functional diversity using solecarbon-source utilisation profiles - a critique. FEMS Microbiol. Ecol. 42: 1-14.

Romero DM, Ríos de Molina MC and Juárez ÁB (2011). Oxidative stress induced by a commercial glyphosate formulation in a tolerant strain of Chlorella kessleri. Ecotoxicol. Environ. Saf. 74: 741-747.

Rutgers M, van't Verlaat IM, Wind B, Posthuma L, et al. (1998). Rapid method for assessing pollution-induced community tolerance in contaminated soil. Environ. Toxicol. Chem. 17: 2210-2213.

Shushkova T, Ermakova I and Leontievsky A (2010). Glyphosate bioavailability in soil. Biodegradation 21: 403-410.

Solomon KR, Anadón A, Carrasquilla G, Cerdeira AL, et al. (2007). Coca and poppy eradication in Colombia: environmental and human health assessment of aerially applied glyphosate. Rev. Environ. Contam. Toxicol. 190: 43-125.

Stefanowicz A (2006). The biolog plates technique as a tool in ecological studies of microbial communities. Polish J. Environ. Stud. 15: 669-676.

Sun YH, Yang ZH, Zhao JJ and Li Q (2012). Functional diversity of microbial communities in sludge-amended soils. Physics Procedia 33: 726-731. 
Vreeken RJ, Speksnijder P, Bobeldijk-Pastorova I and Noij THM (1998). Selective analysis of the herbicides glyphosate and aminomethylphosphonic acid in water by online solid-phase extraction-high-performance liquid chromatographyelectrospray ionization mass spectrometry. J. Chromatogr. A 794: 187-199.

Waiman CV, Avena MJ, Garrido M, Band BF, et al. (2012). A simple and rapid spectrophotometric method to quantify the herbicide glyphosate in aqueous media. Application to adsorption isotherms on soils and goethite. Geoderma 170: $154-158$.

Yu XM, Zhou GF and Xin L (2010). Study on factors influencing the siderophore production of Bacillus subtilis Bs-15 and effects of Bs-15 on disease control and growth promotion of sweet pepper. Chin. J. Pestic. Sci. 12: 135-141.

Yu XM, Ai CX, Xin L and Zhou GF (2011). The siderophore producing bacterium, Bacillus subtilis CAS15, has a biocontrol effect on Fusarium wilt and promotes the growth of pepper. Eur. J. Soil Biol. 47: 138-145.

Zablotowicz RM, Accinelli C, Krutz LJ and Reddy KN (2009). Soil depth and tillage effects on glyphosate degradation. J. Agric. Food Chem. 57: 4867-4871. 